\title{
Chemical Rhizotomy for Relief of Spasticity in Ambulant Patients
}

\author{
P. W. NATHAN,* M.D., F.R.C.P.
}

Brit. med. F., 1965, 1, 1096-1100

In a previous paper (Nathan, 1959) it was stated that when patients who were apparently completely paralysed by upper motor neurone lesions causing spasticity were treated with concentrated solutions of phenol applied around the spinal roots voluntary movements were sometimes uncovered, and it was stated that this technique was being modified so that it might be used for the treatment of spastic patients who can still walk. Since then considerable experience has been gained with modifications of this technique and from experimental studies on cats. The results of this modified treatment are reported here. Films taken before and after treatment are the best way of showing the results, and they were made in all cases; but as they reach only a small number of those who treat these patients it is also essential to report the results in a paper.

Although the series of cases is small, the results obtained show that this kind of chemical rhizotomy is of definite value in the treatment of ambulant spastic patients. Twenty-four patients in whom the aim was to improve their walking were treated; in addition the upper limb was also treated in two patients with hemiplegia.

\section{Results}

An overall view of the results obtained in treating the lower limbs is given in the Table. Half the patients considered themselves to be better, and one-fifth very much better. This is inevitably a condensed way of presenting the patient's opinion. For a patient might be very pleased with the effects of the treatment in stopping pain, while being disappointed that it did not improve his ability to walk as much as he had hoped. He would then have to evaluate the total result achieved according to one of the six categories of the table. Twenty-one out of 23 were rendered less spastic and 16 out of 18 had a reduction in the number and severity of the spasms. This assessment was made by me and the physiotherapist treating the patient. The total for "effect on spasticity" is 23 and not 24 , as one patient was rendered flaccid; this was the patient who thought that she was made much worse, as indeed she was. The totals for "effect on spasms" and "effect on pain" are not 24, as not all patients had these manifestations of spasticity. With regard to the total effect on the patient's ability to move and get about, three were very much better, two were much better, and 11 were better; six showed no change and two were worse.

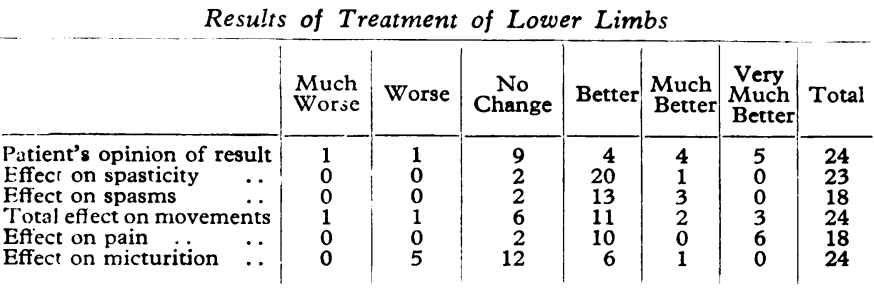

The two important reasons why a total or partial cure of the spasms and a partial relief of spasticity failed to improve the patient's ability to move are the following. The lesion or lesions giving rise to the patient's spastic condition does not affect voluntary movements in all patients to the same degree; in one patient voluntary movements may be severely

* Member of External Scientific Staff, Medical Research Council, National Hospital for Nervous Diseases, Queen Square, London. impaired and in another slightly impaired, and both may show the same degree of spasticity. If there is much actual paresis in addition to the spasticity and spasms, then relief of the latter may not improve the patient's purposeful movements. The second reason is that the treatment may weaken one or more muscles. This is not always a disadvantage; in some cases one of the aims of the treatment may be to weaken a certain muscle or group of muscles that is constantly in activity and is opposing the weaker action of another group. But usually the patient's ability to walk or to go up and down stairs was made worse if muscles were weakened, and then the advantages obtained from a reduction in the spasms and the spasticity were offset by the weakness.

Six patients had all pain totally removed; they have been placed in the category of "very much better" with regard to pain. Ten had the pain relieved; in two there was no effect. Not all patients with spasticity and with spasms have pain; some have a constant aching discomfort with the spasticity, and some have pain with spasms. Patients with pain have placed the relief of pain very high in their estimations of the success of the treatment. Relief of pain has allowed the patients to perform all movements more easily. The stopping of analgesic drugs relieved the constipation that accompanies both upper motor neurone lesions and analgesic drugs, and it has usually resulted in the patients feeling subjectively in better health.

Control of micturition was unaffected in 12 , made worse in five, and improved in seven. One patient found the control of defaecation similarly improved. The five patients in whom micturition was badly affected either experienced increased precipitancy of micturition or increased difficulty in emptying the bladder. The reasons for the improvement in the control of the bladder are thought to be the following. If the bladder tends to empty automatically whenever there is a spasm, then the removal or the diminution of spasms may stop this involuntary emptying. Any diminution in spasticity will help the patient relax the pelvic floor and perineal muscles; as the relaxation of these muscles is a necessary part of micturition and defaecation, this may aid micturition and defaecation.

In two patients injections were performed to reduce spasticity and improve the motility of an upper limb. In one, whose case is reported below, and in whom an injection was also performed for the lower limb, the treatment was a success, although most of the satisfactory effects diminished within a year. In the other there was a greater ability to relax the muscles of the limb, and the muscles showed less constant activity ; but these effects did not help the patient use this limb more easily and efficiently.

Two representative cases are reported. In Case 1 the patient was classified as "very much better." The other (Case 2) is one of the patients who considered himself to be much better.

\section{Case 1}

A spinster aged 61 with disseminated sclerosis was referred on account of pain and spasticity of the lower limbs. She had been dragging the right lower limb for eight years and the left for two years. She had pain in the lower limbs only when she got the spasms. She could manage to walk, keeping her back flexed, her thighs flexed on the pelvis, and dragging both lower limbs very slowly along, the plantar surfaces of the feet never leaving the ground. 
She was given two injections to the nerve roots of the right lower limb. The solution was phenol in glycerol, $5 \%$ on the first occasion and $4 \%$ on the second, and the amount injected was $0.2 \mathrm{ml}$. on both occasions. The roots on which the maximal effect was sought were the second and third lumbar on the first occasion and the fourth and fifth lumbar on the second.

The injections entirely abolished the spasm; and, as pain had been present only with spasms, they removed the pain. All her movements were very much improved, and there was some beneficial effect also in the left lower limb. She was able to raise her feet off the ground when sitting in an upright chair; she could raise her lower limbs from a bed and maintain them extended in the air, and could also abduct them. None of these movements could be done before the treatment. She was able to lie on her abdomen in bedthe first time for many years. When walking she was able to tried to stop it at the second. It may be seen that, before treatment, when she tried to stop the movement, the decline in muscular activity was very gradual and only slight; in fact, following this actual movement, it continued for more than a minute. After treatment, as soon as she tried to stop the movement there was an immediate and great diminution in muscular activity; and the amount of motor-unit activity when she was relaxed was slight.

She had originally come to the National Hospital as she could no longer look after herself alone at home, and the intention had been to send her permanently to a hospital for senile patients. After this treatment she was able to return home and was still living there five years later. Moreover, before the injections she would have to pass urine, or almost have to do so, with every spasm; as the spasms were stopped this disturbance was also removed, and she has remained in normal control of her bladder and bowels.

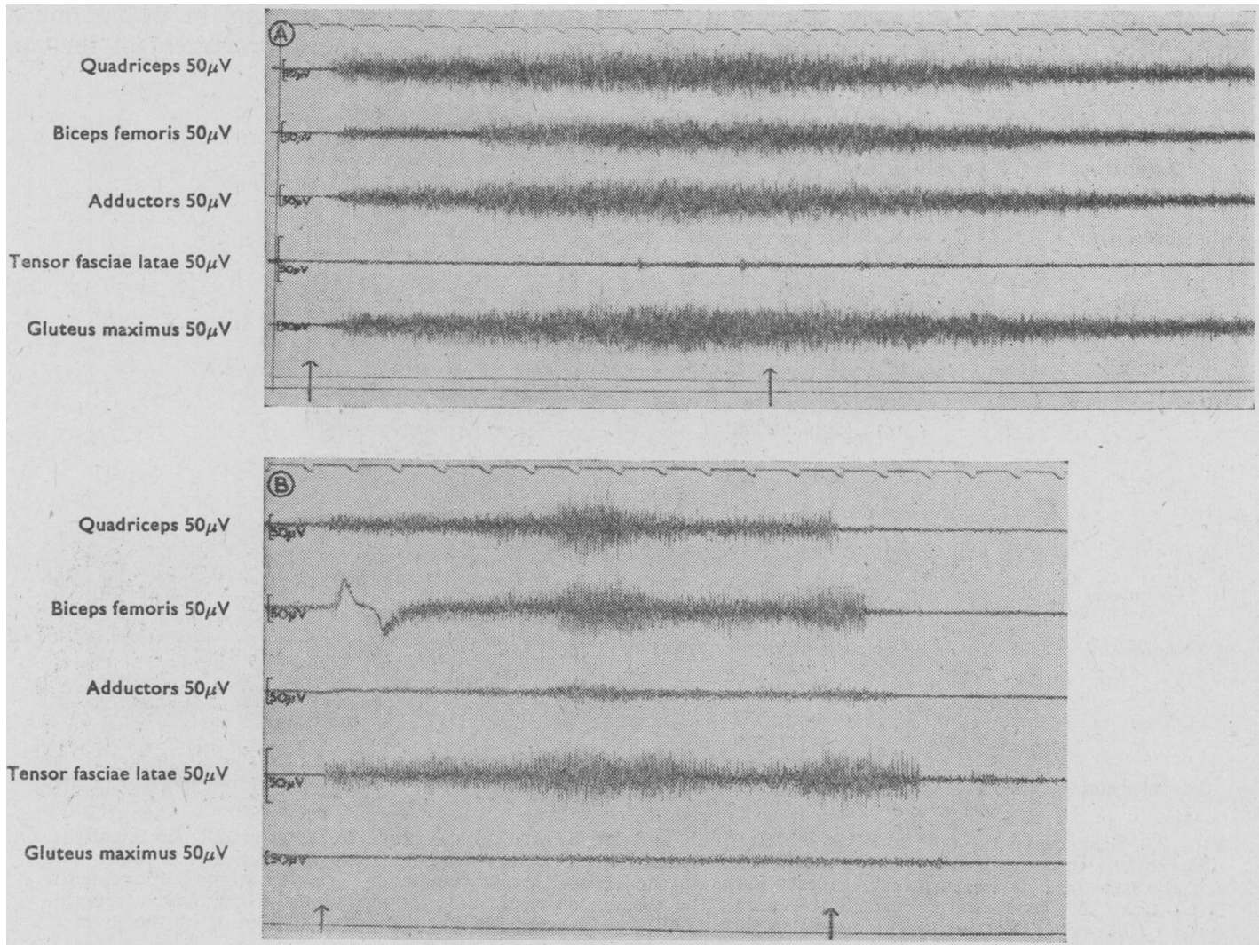

Fig. 1.-Electromyograph of muscles of right thigh before $(A)$ and after (B) the iniections. Between the arrows the patient abducts the two lower limbs; at the second arrow the movement is finished and the patient tries to relax. Before treatment the decline in the activity of motor units is gradual and slow; after treatment there is an abrupt decline in activity and complete relaxation of the adductors and gluteous maximus. Time scale at top of record, 1 sec.

straighten her back so that she could walk in an upright position and not with her back flexed forwards. The injections paralysed the right gastrocnemius muscle; on the other hand, movements returned to the hamstrings and there was much improvement in the glutei. With exercises all movements improved, so that eight months later there was full power in all movements of the thighs on the pelvis and fairly good movements of the ankles and toes.

Electromyographic investigation of many muscles of the lower limbs was carried out before the injection, and on three occasions after them. Before treatment all muscles sampled were in constant activity and the patient could not prevent this. After the treament there were periods of many seconds during which no motor units were active. Before treatment, after a movement a period of one or more minutes would pass before there was any diminution in the activity of motor units; after treatment this period was much shorter. For instance, prior to treatment, flexion of the right lower limb on three occasions took 120,92 , and 135 seconds before any diminution in the activity of all muscles of the limb occurred. After treatment, following flexion of the limb, 12, 15, and 47 seconds elapsed before there was absolutely no activity in the muscles. This is shown in the electromyograms made before and after the injections (Fig. 1). The patient was required to abduct the lower limbs while she was lying on her back. She started the movement at the first arrow and
Unfortunately the disseminated sclerosis progressed inexorably, so that her paralysis and spasticity increased. Yet five years and a half later she still retained certain benefits from the treatment in that she was free from spasms and therefore had adequate control of micturition, and she was also free from pain, for which she felt most grateful.

\section{Case 2}

This patient, a man aged 59, had several cerebral thromboses. He was referred on account of "thalamic pain" in the left limbs. Originally, after the first important thrombosis, he had had monoballism of the left upper limb; but this had largely passed off, leaving some abnormal movements when he adducted the limb, and spasticity affecting mainly the adductors and flexors. In the lower limb the pain was more severe and constant; some spasms and much spasticity were present. The patient seemed to be unusually indifferent to his state; he made no attempt to improve the movements of his left limbs and was apprehensive of anyone else trying to do so.

He was given an injection of $0.5 \mathrm{ml}$. of phenol in glycerol $5 \%$; the roots on which a maximal effect was sought were the fourth and fifth lumbar. After the injection there was a most striking effect 
on the patient's personality. This led us to conclude that he had previously been in a state of reactive depression, and that his tendency to cry had been due to this and not to damage to cerebral tissue as had at first been thought. He ceased to be over-emotional and became quite cheerful, started to help himself to get up and walk about again, and gave up his previous attitude of hopelessness.

When he came to hospital he was unable to walk. On returning home he could walk about his house and garden, and went for accompanied walks in the street. He could still do so three years after the injection. The full control of his bladder was slightly affected, so that to micturate he had to press on his abdominal wall with his hands and massage it towards the pubis.

\section{Comments on Results}

The main features of the state of spasticity are that a great number of muscles are active, that they are active at times when adequate co-ordination for posture and movement requires that throughout but fixes the knee-joint at the start of each flexion and extension of the foot. Further, the activity in the quadriceps, tibialis anterior, and gastrocnemius ceases in an almost normal manner at the end of each contraction; it does not continue, as it did before, making the start of the next opposite movement almost impossible.

Another result of the treatment is the reduction in spasticity and spasms of the muscles of which the nerve roots were not purposely treated. In one or two patients this other improyement has been so pronounced that the reduction in the spasticity of the muscles of their backs was more important to them than that of the lower limb actually treated; it allowed them to sit in chairs, which they had been unable to do before. Others found relief from having the spasticity of the abdominal wall lessened, although the treatment had not been designed to do this. In most patients in whom one lower limb was treated there was some improvement of the contralateral limb.

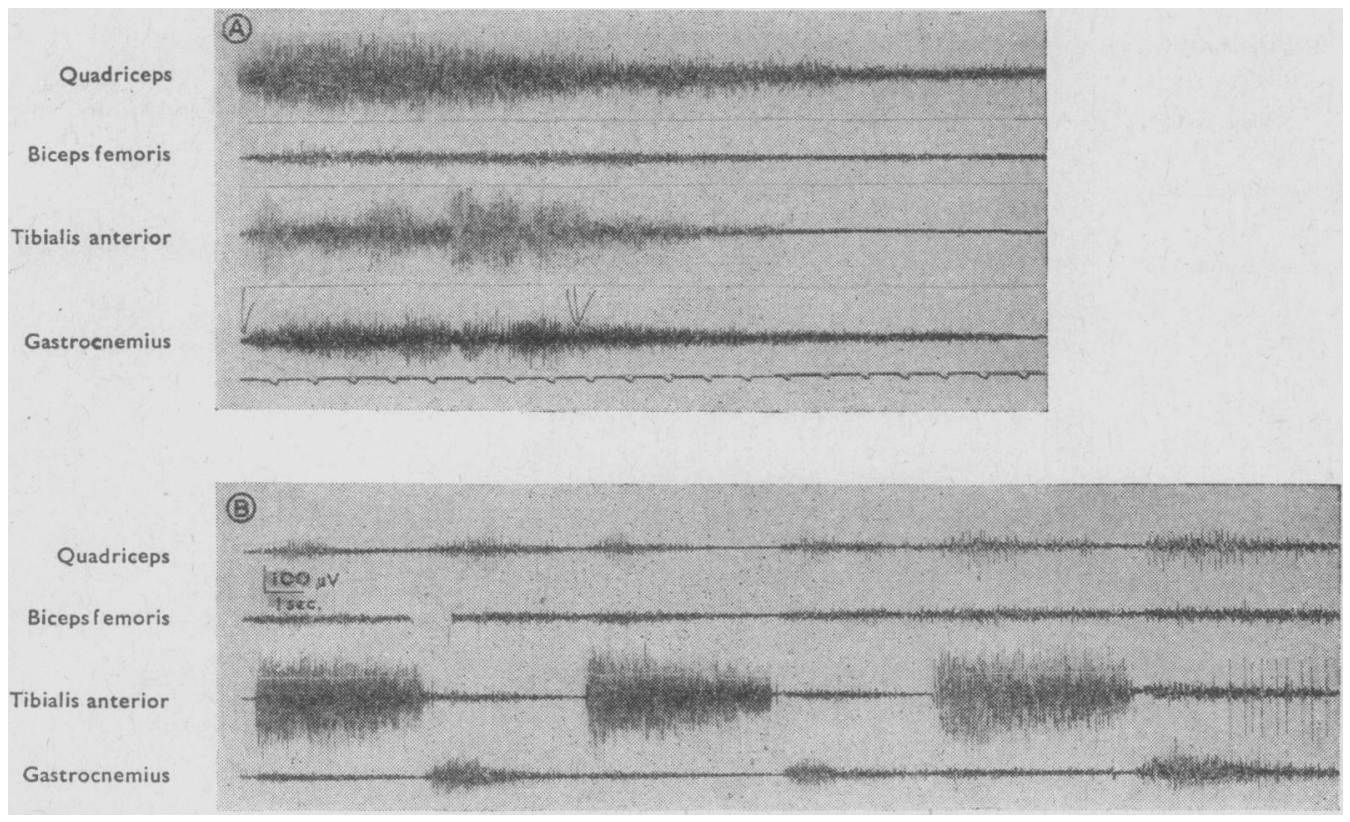

Fig. 2.-Electromyograph of muscles of left thigh and leg before (A) and three weeks after (B) the injections. The patient is carrying out plantar flexion and extension. Before treatment all muscles are brought into activity and there is no reciprocal innervation. At the second arrow the patient tries to stop all movements. activity and there is no reciprocal innervation. At the second arrow the patient tries to stop all movements.
After treatment reciprocal innervation is seen; the tibialis anterior and gastrocnemius are activated reciproAfter treatment reciprocal innervation is seen; the tibialis anterior and gastrocnemius are activated recipro-
cally. The quadriceps no longer contracts throughout the movements but fixes the knee-joint at the start of cally. The quadriceps no longer contracts throughout the movements but
each flexion and extension of the ankle.

they should be inactive, and that posture and movement themselves bring unwanted muscles into activity. During electromyography one can see clearly how muscles continue to contract after the desired movement has been carried out ; some activity of motor units may continue for minutes or even until the patient next goes to sleep, when relaxation occurs. As muscles either do not relax or they relax inadequately, the next movement is greatly impeded, particularly as in walking each subsequent movement is the opposite of the preceding one. After chemical rhizotomy there is a quicker return to inactivity of motor units following movement. Accompanying this there is a return to normal reciprocal innervation.

A typical electromyogram showing these features is presented in Fig. 2. The patient is attempting plantar flexion and extension of the foot alternately. Before treatment a great amount of activity is induced in all muscles; there is no reciprocal innervation. When the patient tries to replace one movement by the opposite movement, no decrease in the activity of the antagonists occurs: all muscles continue to contract. All that she can do is to increase the continuous activity of the flexors and extensors alternately. After treatment reciprocal innervation is clearly seen: the tibialis anterior and gastrocnemius are innervated alternately; and the quadriceps does not contract

\section{Durability of Improvement}

Although some of these results have been reported to medical societies in this country and in the United States, publication has been delayed until a follow-up period has been adequate. Patients in whom the treatment was a failure were not followed up. Of the 13 in whom it was a success, the length of follow-up has been five and a half years in two, four and a half years in four, two years in three, 18 months in three, and six months in one. The question whether the benefit obtained is permanent cannot be answered simply ; for the patients who were treated were not suffering from stationary pathological conditions.

The results in those patients who were very much better, much better, or better for various periods after treatment are given here. Three have maintained all the ground gained for four to five years, one has maintained it for three years, one for 15 months, and one for six months since the treatment. One patient with disseminated sclerosis retained all the improvement for 11 months; then she got a further severe attack of disseminated sclerosis, making her for a time completely paraplegic. She gradually recovered from this attack, but her lower limbs remained bereft of a great deal of purposive movements, so that she could hardly move her spastic limbs; this state was 
worse than before the injections. Two other patients with disseminated sclerosis got a further attack of the disorder one year after the treatment, and have been left worse than they were before. Another patient with disseminated sclerosis retained the benefit for four years, but the disease has slowly become more severe and she is now unable to walk. One patient who had severe paraparesis following meningomyelitis maintained the improvement in her legs for two years, but then could not walk more than a few steps because of weakness of the upper limbs, with consequent inability to use walkingsticks, and because of heart failure. One patient who had amyotrophic lateral sclerosis had become so paralysed with the progress of his disorder by four months after the treatment that he was again unable to do more than walk round his bed. Another-the patient with the thalamic syndrome following cerebrovascular accidents-retained the benefit to the upper limb for one year and so far has retained that to the lower limb for five years.

\section{Technique of Procedure}

The solutions which have been tried were phenol in glycerol, $2 \%, 4 \%$, and $5 \%$, and phenol in iophendylate (Myodil) $10 \%$, $15 \%$, and $20 \%$. The solution that has so far been found most useful is phenol in iophendylate $10 \%$.

The technique is essentially the same as that previously described (Nathan, 1959). The injection is best done in the radiology department, as a good tilting-table must be used, and it is advisable to take a radiograph of the spine after the spinalpuncture needle has been inserted in order to be sure of the vertebral level of the point of the needle. As the solution is heavy the patient is placed in such a position that the roots to be treated are at the lowest point. The patient lies curled up on his side as for a lumbar puncture, with the vertebrae of the roots to be treated at the lowest point. The needle is inserted so that its point is nearest to the root that it is most important to treat. After the needle has been inserted the patient's position is altered, the plane of his back being tilted backwards towards the table so as to make an angle of about 75 degrees with the top of the table. The purpose of repositioning the patient slightly more posteriorly is to try to produce the maximal effect of the phenol solution on the posterior roots. Before the solution is injected the needle is withdrawn slightly and turned so that the hole of the bevelled tip faces downwards towards the table. The total amount given during one injection has varied between 0.2 and $1 \mathrm{ml}$. It is best to inject about $0.2 \mathrm{ml}$. in most cases and see what effect is obtained during the quarter of an hour when the patient is lying on his side. At the end of that time, if the lower limb is still very spastic and voluntary movements have been only slightly improved, up to another $0.25 \mathrm{ml}$. may be injected; if the limb is far looser and if there is any weakness no more should be injected.

In order to find out upon which posterior roots the phenol solution is acting, the sensibility of the skin must be tested by pricking. This examination must start with the sacral dermatomes, over the lowermost buttock. If loss of sensibility is found over this region the tilt on the table is altered, so that the flow of solution is less caudal. Provided the tilt is altered within two minutes no damage to the sacral-nerve supply of the bladder and anus will have been done. It will be found that within 20 minutes the loss of sensibility in this region will have gone off. When both lower limbs need to be treated, as they usually do, it is probably advisable to do this on separate occasions.

\section{Discussion}

This work was based on certain theoretical premises that were eventually found to be wrong. It was begun with the belief that the solutions of phenol probably destroy the smaller nerve fibres selectively. This view was held as Maher (1955) had shown that the solutions can permanently stop chronic severe pain, and it is known that pain sensibility is subserved by the delta group of myelinated fibres and the non-myelinated $\mathrm{C}$ fibres. And it was shown by Iggo and Walsh (1960) and Nathan and Sears (1960) that when the solutions act as local anaesthetics they block small fibres rather than large. The intention was to destroy selectively the nerve fibres of the anterior roots that supply the intrafusal muscle fibres. It was reckoned that this would greatly reduce the stretch reflexes of the muscles of which the anterior roots had been treated without damaging the nerve fibres to the muscles themselves, and so the muscles would be less spastic and would still be available for movements.

During the ensuing years those of us working here on this problem have come to the conclusion that when the solution is used in such a way as to destroy nerve fibres it does so in a non-selective manner, destroying some nerve fibres of all types and sizes. Evidence showing this has been published by Smith (1964) and by Nathan, Sears, and Smith (1965). We then found ourselves in the position of carrying out a therapeutically successful procedure and of having no explanation to account for its success. Eventually a hypothesis was arrived at. This is supported by evidence presented elsewhere (Dimitrijević and Nathan, 1965). Briefly this hypothesis is as follows. In spasticity of the spino-muscular type there is overactivity of motor units, and this overrides the built-in spinal patterns of motor co-ordination. The overactivity results in an inability to grade and to separate movements, difficulty in stopping one movement so as to start another, difficulty in restricting movements to a limb and in performing separate movements. We suppose that the overactivity of the motoneurones constituting the final common paths is the outcome of an excessive inflow to these neurones, whether it originates within the spinal cord, within the brain, or in the inflow through the posterior nerve roots. It follows from this that any reduction in the inflow will reduce the excessive activity of the motoneurones and will allow the basic patterns of co-ordination which are '. tent to become manifest. Thus our previous qualitative hypot.. sis has been replaced by a quantitative one; and the previous aim of destroying selectively the fusimotor fibres of the anterior roots has been shifted to destroying a quantity of fibres of the posterior roots.

\section{Selection of Patients}

The main problem in treating cases of spasticity with this method is to select the right patients. Probably some of the patients chosen should not have been treated and others may have been wrongly refused; only from treating patients was experience gained on the results that could be achieved and on the kind of patient who would benefit from the treatment. The ideal patient is one with considerable pain who has retained a great deal of voluntary movement which is greatly impeded by involuntary spasms and spasticity, and whose micturition and defaecation are also upset by the spasms and spasticity. Patients should be treated before contractures develop; but it is probably inadvisable to use this destructive procedure if any spontaneous recovery is still likely to occur. The impression has been gained that if the patient is ataxic he will not be improved and may be made worse.

To find the patient most likely to benefit from this treatment it is necessary to try to discover if he has retained the ability to carry out voluntary movements, so that once the spasticity has been reduced he will be able to move well. There is no simple way of being sure of this. For it is difficult to determine whether a muscle is available for voluntary movements when its activity is constantly overcome by that of its antagonists and when all attempts at moving are overcome by spasticity and 
spasms. Moreover, muscles are used in different ways in different movements. A muscle which a patient cannot contract on making a voluntary effort may contract with a reflex movement. A muscle that cannot be activated with one voluntary movement, say extension, may be activated voluntarily with another voluntary movement, say abduction. After spasticity had been successfully reduced, I sometimes realized that I had been previously misled in two directions: muscles which I thought could be voluntarily contracted were working only in conjunction with a reflex spasm; and muscles which apparently could not be voluntarily activated turned out to be under voluntary control once spasticity and spasms had been subdued.

It is easier to achieve an improvement in the lower limb than in the upper limb. Upper limbs have to be used for fine and skilled movement. Props with some degree of mobility may be all that can be achieved for the lower limbs, yet these are a great improvement on a total inability to move.

Certain aspects of spasticity may be useful in paraparesis. On the whole, extensor tone is desirable and flexor tone is not ; yet an excessive involuntary activation of the extensors can impede the flexors and almost stop the patient walking. In some patients, although it is rare, the pressure of the foot on the ground induces extensor tone, and this helps or enables them to stand and walk; as the physiotherapists so aptly put it: " he walks on his spasms." Flexor spasms coming on suddenly may drop the patient to the ground, and clonus can be so troublesome that it throws the patient off his feet. Excessive adductor tone or spasm is always undesirable ; it prevents walking. Walking in a normal manner is not necessarily the aim of treatment. For instance, a normal subject uses his hamstring muscles and the extensors of his thighs to keep himself upright ; a patient with poor control and co-ordination of these muscles can lean forward on two tripods and make use of his upper limbs, his back, and his balance to achieve this. If a patient $r_{\text {ds }}$ a weak ilio-psoas he may be allowed to flex his thigh with his rectus femoris or to circumduct the limb. Contrary to what $\operatorname{mig}^{\circ} \therefore$ be thought, patients are usually inept at making use of the : vements they have retained; a good physiotherapist can teach them a lot.

To try to find out if the patient will benefit from this treatment or not, it is important to examine him on many occasions and to do so when he is up and walking, lifting his feet up and down stairs, getting into and out of chairs, and performing movements such as marking time and abducting the legs. When the patient is lying or sitting it is important to obtain relaxation of the limb before getting him to perform movements. The patient's movements and posture should be examined to find out if there is one muscle or muscle group which is constantly active. Electromyography is most useful for this. If it is found to be so, one must decide whether the weakening of that muscle or muscle group would improve the patient's ability to stand and walk, or not.

Finally, a sphygmomanometer test has been found useful. If ischaemia is induced in the limb for an adequate length of time there follows a decrease in the excitability of the muscle receptors, so that stretch reflexes diminish. A large, wide sphygmomanometer cuff is inflated to a pressure exceeding the systolic pressure, and left on the upper thigh for 20 to 25 minutes. Unfortunately, unlike a cuff on the arm, this is painful and many patients will not tolerate it. The consequences of this ischaemia are a relatively early reduction of spasticity of the muscles distal to the cuff and a later reduction in that of muscles proximal to the cuff and even of the contralateral limb. Experience has shown that if the muscles distal to the cuff are improved the chemical rhizotomy is likely to be successful ; moreover, if the muscles proximal to the cuff are also improved, this improvement may also be achieved by the rhizotomy.

Some patients have already lost so much voluntary movement that, although the reduction in spasticity and still more the reduction in spasms may help them, their ability to walk or even to stand is not improved. Other patients stand and move by inducing extensor spasms. Whether they will be able to move as well when extensor tone is lessened and extensor spasms are stopped is difficult to ascertain. When the joints are fixed so that the limb is appreciably shortened it is useless to improve the movements, unless surgical procedures are also provided to correct the deformity or the patient is given surgical appliances, such as built-up boots or toe-springs.

\section{Summary}

A form of treatment of ambulant patients with spastic lower limbs by means of the intrathecal injection of phenol in glycerol or iophendylate is described.

Half the patients treated thought that their condition was much improved. A considerable reduction in spasticity and in the occurrence of spontaneous spasms was achieved; but weakness was sometimes increased.

In two-thirds of the patients there was much improvement in the ability to walk. In successfully treated cases the patients who had been unable to walk more than a few steps have been enabled to walk several hundred yards.

The pain associated with spasms and/or spasticity was reduced or abolished; this result was considered by the patients to be one of the main benefits of the treatment.

This form of treatment has both improved and impaired patients' control of micturition.

Certain theoretical aspects of the treatment are discussed.

\section{REFERENCES}

Dimitrijević, M. R., and Nathan, P. W. (1965). In preparation.

Iggo, A., and Walsh, E. G. (1960). Brain, 83, 701.

Maher, R. M. (1955). Lancet, 1, 18.

Nathan, P. W. (1959). Ibid., 2, 1099.

- and Sears, T. A. (1960). f. Physiol. (Lond.), 150, 565.

- - and Smith, M. C. (1965). 7. neurol. Sci., 2, 7.

Smith, M. C. (1964). Brit. F. Anaesth., 36, 387 